Nouvelles perspectives en sciences sociales

Revue internationale de systémique complexe et d'études relationnelles

\title{
Complexité dans une langue isolante : exemple du vietnamien
}

\section{Danh Thành Do-Hurinville}

Volume 9, numéro 1, novembre 2013

Sur le thème : linguistique et complexité

URI : https://id.erudit.org/iderudit/1024044ar

DOI : https://doi.org/10.7202/1024044ar

Aller au sommaire du numéro

\section{Éditeur(s)}

Prise de parole

\section{ISSN}

1712-8307 (imprimé)

1918-7475 (numérique)

Découvrir la revue

\section{Citer cet article}

Do-Hurinville, D. T. (2013). Complexité dans une langue isolante : exemple du vietnamien. Nouvelles perspectives en sciences sociales, 9(1), 239-267. https://doi.org/10.7202/1024044ar

\section{Résumé de l'article}

Toutes les langues sont aussi complexes les unes que les autres, mais le degré de complexité peut ne pas être similaire dans un domaine donné. Le présent article vise à examiner l'hypothèse compensatoire (selon laquelle les langues isolantes sont moins complexes que les langues flexionnelles du point de vue morphologique, mais peuvent être, par compensation, plus complexes du point de vue syntaxique et sémantique) en passant au crible la grammaire du vietnamien, langue isolante, dont la complexité est examinée à trois niveaux : morpho-phonologique, syntactico-sémantique et discursif. 


\title{
Complexité dans une langue isolante : exemple du vietnamien
}

\author{
Danh Thành Do-HurinviLle \\ Institut national des langues et civilisations orientales, \\ SeDyl UMR 8202-CNRS
}

\section{Problématique}

Ta notion de complexité est explorée dans plusieurs types de Lsciences (sciences naturelles, sciences de l'information, sciences humaines), mais sa définition semble connaître des nuances selon ces différentes sciences. Dans sa thèse de doctorat, Nathalie Glaudert ${ }^{1}$ précise que " La complexité est un problème très ancien, aussi vieux que l'analyse des systèmes quels qu'ils soient, mais aussi un problème très actuel, notamment dans l'analyse des langues [...]». D'après Matti Miestamo, Dahl ${ }^{2}$ et bien d'autres linguistes, les travaux consacrés à la complexité en linguistique foisonnent aussi bien en linguistique typologique et comparative que dans l'acquisition du langage.

$1 \quad$ Nathalie Glaudert, La complexité linguistique : essai de théorisation et d'application dans un cadre comparatiste, Thèse de doctorat, Université de la Réunion, 2011, p. 23.

2 Matti Miestamo, "Grammatical Complexity in Cross-Linguistic Perspective ", dans Matti Miestamo, Kaius Sinnemäki et Fred Karlsson (dir.), Language Complexity: Typology, Contact, Change, Amsterdam, Benjamins, 2008, p. 23; Östen Dahl, The Growth and Maintenance of Linguistic Complexity, Amsterdam, Benjamins, 2004. 
Glaudert souligne que « [1]'analyse du complexe est attrayante car le complexe est problématique, mystérieux, parfois même incompréhensible à première vue. C'est en cela que le caractère complexe des éléments est souvent assimilé, à tort, à de la supériorité $^{3}$ ". Pour étayer son point de vue, elle a cité Wouter Kusters ${ }^{4}$, qui a mis en garde contre toute tentative du rapprochement de la complexité d'une langue à l'état de civilisation du pays où on parle cette langue :

De nombreux linguistes mais aussi non linguistes considèrent la propriété " d'être complexe » comme égale à « avoir une grande valeur (dans un sens cognitif, social ou culturel) ». En effet, la musique aux harmonies complexes ou les textes littéraires aux interprétations complexes sont souvent plus appréciés que leurs opposés. De telles considérations esthétiques supportées par des raisons chauvinistes ont mené beaucoup de linguistes du XIX ${ }^{e}$ siècle à supposer que les langues comme le latin, le sanskrit et l'allemand seraient plus complexes, et que les langues plus complexes sont liées à des cultures plus complexes et plus prestigieuses (traduit en français par Glaudert) . $^{5}$

À ce sujet, Edward Sapir a clairement dissocié l'évolution de la langue de celle de la culture et des mœurs :

Et je ne puis croire non plus que les mœurs et le langage soient en rien dépendants l'un de l'autre. Les mœurs sont le résultat de ce qu'une société fait et pense, le langage est la manifestation particulière de la pensée; il est difficile de discerner quelles relations de cause à effet peuvent exister entre une sélection des produits de la connaissance (les mœurs ne sont autres qu'un choix que la société opère dans les comportements) et la façon particulière dont se traduit cette connaissance. L'évolution des mœurs, autre façon de nommer l'histoire, est une suite

$3 \quad$ Nathalie Glaudert, op. cit., p. 22.

4 Wouter Kusters, "Complexity in Linguistic Theory, Language Learning and Language Change ", dans Matti Miestamo, Kaius Sinnemäki, Fred Karlsson (dir.), Language Complexity: Typology, Contact, Change, Amsterdam, Benjamins, 2008, p. 3.

5 Jean-Baptiste Coyos ( La langue basque selon Gustave Guillaume : quelques commentaires d'un point de vue structuraliste fonctionnaliste ", dans les Actes $d u X X^{e}$ Colloque international de psychomécanique du langage, Oloron-SainteMarie (France), 2004, p. 8) note ceci à propos de Guillaume : "Où par contre, on ne peut plus suivre Guillaume, c'est quand il lie type de langue et histoire des langues, histoire de la pensée humaine et même état de civilisation" (p. 8). 
complexe de changements survenus dans les comportements de la société, ses acquisitions, ses pertes, les transferts de ses centres d'intérêt et de ses rapports. L'évolution du langage ne s'occupe nullement des changements du contenu mais seulement des changements de la forme. Il est possible, en pensée, de changer chaque son, chaque mot, chaque concept concret du langage, sans changer en rien sa signification intrinsèque, exactement comme l'on peut verser dans un moule immuable de l'eau, du plâtre ou de l'or fondu. Si l'on arrive à démontrer que les mœurs ont pour base un plan inné, qu'elles évoluent suivant une courbe donnée, nous avons alors un terme de comparaison avec une possibilité de les relier entre eux. Mais jusqu'à ce que l'on ait découvert ces systèmes purement abstraits qui pourraient régir la culture en général, nous ferons bien de tenir le langage et les mœurs comme non comparables et sans lien dans leur évolution. Tous les essais pour relier certains types morphologiques à certains stades culturels ou à un certain ensemble de mœurs sont vains; semblable rapprochement est futile; le plus petit coup d'œil vérifie notre théorie sur ce point : des types de langages simples, ou complexes, d'une variété infinie, se retrouvent à n'importe quel échelon culturel; lorsqu'il s'agit de la forme linguistique, Platon a pour égal le berger macédonien et Confucius ne se distingue pas du sauvage d'Assam ${ }^{6}$.

Ramat Paolo est convaincu que " aucune langue connue le long des 6000 ans de notre documentation ne peut être considérée primitive, au sens qu'elle reflète un état primitif de la communication linguistique humaine. En effet, comme on va le voir, 'non complexe' n'équivaut pas à 'primitif' pas plus que 'complexe' n'équivaut à 'évolué'7 ".

Selon Hockett, Aitchison, McWhorter, Dahl, Shosted et Kusters ${ }^{8}$, toutes les langues sont aussi complexes les unes que les

6 Edward Sapir, Language: An Introduction to the Study of Speech, New York, Harcourt, Brace (Le langage. Introduction à l'étude de la parole, traduit de l'anglais par Guillemin), 1921, p. 154.

7 Ramat Paolo, "De la complexité des langues. À propos de l'axiome 'ALEC' (All Languages are Equally Complex) ", communication à la journée d'étude La nature de la complexité linguistique, organisée par Claire Martinot, 2 et 3 juillet 2012, Maison de la recherche, Paris.

8 Charles Francis Hockett, A Course in Modern Linguistics, New York, Macmillan, 1958; Jean Aitchison, Language Change: Progress or Decay? Cambridge, Cambridge University Press, 1991; John Hamilton McWhorter, "The world's simplest grammars are creole grammars ", Linguistic Typology, $\mathrm{n}^{\circ}$ 5, 2001, p. 125-166; Östen Dahl, op. cit.; Ryan Shosted, "Correlating 
autres, ce qui corrobore la position de Sapir :

L'affirmation que toutes les langues du monde ont la même complexité (en anglais 'All Languages are Equally Complex') a representé jusqu'à des années récentes presque un dogme que tous les linguistes posaient à la base de leurs comparaisons interlinguistiques. À l'intérieur de la théorie de la Grammaire Universelle du générativisme c'était quasiment un axiome?

\section{Partageant ce point de vue, Glaudert ajoute que :}

De façon absolue, il n'existe pas de langue simple ni de langue complexe. Il a été démontré que les différentes composantes d'une langue sont régies par des " compromis " entre simplicité et complexité (FenkOczlon et Fenk 2008), qui visent à garder un système linguistique globalement efficace et économique. C'est ce que Gil ${ }^{10}$ nomme "l'Hypothèse de la Compensation ": en d'autres termes, un équilibre délicat entre simplicité et complexité parmi les différentes composantes d'une langue (et non à l'intérieur de celles-ci). Cela nous amène à postuler que toutes les langues sont complexes de façon à peu près égale ${ }^{11}$.

Analysant trois langues isolantes de l'Asie (le hmong, le chinois et le thaï), Elizabeth Riddle ${ }^{12}$ adhère à l'hypothèse de la compensation (Compensation Hypothesis), selon laquelle si certaines langues (notamment les langues isolantes) sont plus simples morphologiquement, elles sont, par compensation, plus complexes du point de vue syntaxique et sémantique. Pour sa part, Walter Bisang ${ }^{13}$ évoque la complexité cachée (bidden complexity)

complexity: A Typological Approach ", Linguistic Typology 10, 2006, p. 1-40; Wouter Kusters, op. cit.

9 Ramat Paolo, op. cit., (p. 1).

10 Toutefois, Gil cherche à démontrer le contraire de cette hypothèse. Voir David Gil, "How Complex are Isolating Languages ", dans Matti Miestamo, Kaius Sinnemäki, Fred Karlsson (dir.), Language Complexity: Typology, Contact, Change, Amsterdam, Benjamins, 2008, p. 109-131.

11 Nathalie Glaudert, op. cit., p. 22.

12 Elizabeth M. Riddle, "Complexity in Isolating Languages: Lexical Elaboration Versus Grammatical Economy ", dans Matti Miestamo, Kaius Sinnemäki, Fred Karlsson (dir), Language complexity: Typology, Contact, Change, Amsterdam, Benjamins, 2008, p. 133-151.

13 Walter Bisang, "On the Evolution of Complexity - Sometimes Less is More in East and Mainland Southeast Asian ", dans Geoffrey Sampson, David Gil, Peter Trudgill (dir.), Language Complexity as an Evolving Variable, Oxford, Oxford University Press, 2009, p. 34-49. 
quant au fonctionnement de ces types de langues.

Dans cet article consacré à l'analyse de la complexité en vietnamien, j'examinerai le point de vue de Riddle sur la complexité dans les langues isolantes, et tenterai de montrer les limites de l'hypothèse compensatoire.

\section{Complexité en vietnamien}

Afin d'avoir une vue globale sur la complexité du fonctionnement du vietnamien, j'étudierai les composantes de cette langue aux niveaux morpho-phonologique, syntaxique et sémantique et discursif.

\subsection{Complexité au niveau morpho-phonologique}

On soulignera la structure des syllabes et notamment la naissance des six tons dont dispose le vietnamien et la réduplication des mots.

Miestamo ${ }^{14}$ distingue, d'une part, la complexité absolue (qui relève de la théorie et de la position objective) et, d'autre part, la complexité relative (qui appartient à la position subjective de l'apprenant). Une langue comme le vietnamien, pourvue de six tons, doit être théoriquement et objectivement plus complexe, à ce sujet, que des langues voisines comme le chinois ou le thaï, qui en comptent respectivement quatre et cinq, ou que des langues indo-européennes comme le français et l'italien qui en sont dépourvus.

\subsubsection{Structure des syllabes et naissance des tons}

En vietnamien, les syllabes se comportent comme des unités de base : la grande majorité d'entre elles sont des morphèmes, dont une proportion substantielle a un statut de mot, ce qui conduit à penser que le vietnamien est une langue monosyllabique. Cependant, Phu Phong Nguyên ${ }^{15}$ précise que tous les mots vietnamiens ne sont pas monosyllabiques, et qu'il existe une

\footnotetext{
14 Matti Miestamo, op. cit., p. 24-26.

15 Phu Phong Nguyên, Le syntagme verbal en vietnamien, Paris, Mouton, La Haye, 1976, p. 17.
} 
proportion non négligeable de mots polysyllabiques. On peut dire que le vietnamien est une langue syllabique.

Une syllabe vietnamienne comporte au maximum les cinq éléments suivants : le ton, la consonne initiale, le glide, le noyau et la consonne finale. Parmi ces éléments constitutifs, seuls le ton, la consonne initiale et le noyau sont obligatoires, le glide et la consonne finale étant facultatifs. Examinons la syllabe toán, morphème lexical signifiant " mathématiques ", qui s'analyse comme suit : la consonne initiale (notée $\mathrm{C}_{1}$ )/t/ (orthographiée ' $\mathrm{t}$ '), le glide (noté $/ w /$ ) (orthographié 'o'), le noyau vocalique (noté V) /a/ (orthographié 'a'), la consonne finale /n/ (orthographiée 'n'), et le ton (noté $\mathrm{T}$ ), qui est le ton montant (accent aigu en orthographe). La structure de ce lexème est la suivante : $\left(\mathrm{C}_{1}\right)(\mathrm{w})$ $\mathrm{V}\left(\mathrm{C}_{2}\right)+\mathrm{T}$.

La langue vietnamienne dispose, depuis près de dix siècles, de six tons : le ton égal (ou ton 1), le ton descendant (ou ton 2), le ton montant (ou ton 3), le ton descendant montant (ou ton 4), le ton montant glottalisé (ou ton 5) et le ton tombant glottalisé (ou ton 6).

Dans cette langue, le ton est une marque phonologique permettant de différencier des morphèmes de structure segmentale identique. Le ton remplit donc une fonction distinctive (1). Cela corrobore la remarque de Dahl au sujet des langues à tons : " tone distinguishing monosyllabic lexical items or encoding morphosyntactic distinction ${ }^{16}$ ".

(1) $M a$ (ton égal ou ton 1) est un lexème signifiant "fantôme ".

Mà (ton descendant ou ton 2) est une conjonction correspondant à " mais ".

Má (ton montant ou ton 3) est un lexème signifiant " maman " ou " joues".

Mả (ton descendant montant ou ton 4) est un lexème signifiant " tombe ». Mã(ton montant glottalisé ou ton 5) est un lexème signifiant, selon le contexte, " apparence ", " plumage ", " cheval ", " code ".

$M a$ (ton tombant glottalisé ou ton 6) est un lexème signifiant " jeune pousse de riz » ou « recouvrir un métal d'une couche d'un autre métal ».

16 Östen Dahl, op. cit., p. 108. 
Georges Haudricourt souligne que le vietnamien ancien, le thaï ancien, le chinois archaïque et le miao-yao commun étaient des langues atonales au début de notre ère, et que "l'apparition des tons engendrée par les modifications des consonnes finales et initiales a dû se produire parallèlement dans les quatre langues, sous l'influence culturelle du chinois, influence dont nous avons le témoignage par les emprunts ${ }^{17}$ ». De nos jours, le vietnamien est riche de six tons, dont trois sont apparus vers le $6^{\mathrm{e}}$ siècle et les trois autres vers le $12^{\mathrm{e}}$ siècle. En d'autres termes, le système tonal vietnamien se complexifie au fil de plusieurs siècles : il s'agit donc d'un phénomène diachronique. La naissance des tons se produisit peu à peu corrélativement à une érosion phonétique (chute de consonnes finales); on constate une transformation de consonnes initiales (de sourdes en sonores), comme dans le tableau 1.

Tableau 1 (emprunté à Haudricourt ${ }^{18}$ ).

\begin{tabular}{|l|l|l|l|}
\hline Début de notre ère & $6^{\mathrm{e}}$ siècle & $12^{\mathrm{e}}$ siècle & De nos jours \\
\hline pas, pah & pà & pả & bả \\
slas, hlah & hlà & lả & là \\
\hline
\end{tabular}

En d'autres termes, on peut dire qu'une simplification phonétique est compensée par une complexification des tons : slas ou hlah composés de quatre lettres sont devenus de nos jours, là, ne comportant que deux lettres, mais doté d'un ton.

\subsubsection{Réduplication des mots}

Riddle $^{19}$ souligne que les langues isolantes (ou langues analytiques), dépourvues de flexions, sont considérées comme plus simples que les langues synthétiques, mais que ces langues isolantes sont dotées d'expressions lexicales, dont la formation est bien plus complexe que celle qu'on peut rencontrer dans des

\footnotetext{
17 Georges Haudricourt, "De l'origine des tons en vietnamien ", Journal Asiatique, no 242, 1954, p. 82.

18 Georges Haudricourt, op. cit., p. 81.

19 Elizabeth M. Riddle, op. cit., p. 133.
} 
langues synthétiques comme le polonais et l'anglais. La réduplication des mots en vietnamien permet d'étayer ce point de vue.

La réduplication, qui est une caractéristique essentielle des langues isolantes du Sud-Est de l'Asie, se rencontre très fréquemment en vietnamien (conversation, littérature, poésie...). Il s'agit de rédupliquer une partie du mot (réduplication partielle) ou le mot en entier (réduplication intégrale).

(2) bâng khuâng (être mélancolique), bẽ lẽen (être rougissant), bì̀n chî̀n (être fébrile), cằn nhằn (grogner), chơi vơi (être isolé, au gré des circonstances), etc.

L'exemple (2) illustre une réduplication partielle, puisque dans les mots ci-dessus seule la rime (en caractères gras), composée du noyau vocalique, de la consonne finale et du ton, est rédupliquée.

$$
\begin{array}{lll}
\text { (3) } \quad \text { sáng }(\text { matin }) \rightarrow & \text { sang sáng } & \text { (au petit matin) } \\
\text { (4) } \quad \text { sáng }(\text { matin }) \rightarrow & \text { sáng sáng } & \text { (tous les matins) }
\end{array}
$$

L'exemple (3) représente une réduplication quasi-intégrale, alors que (4) montre une réduplication intégrale. La complexité réside dans le jeu des tons. En (3), les tons des deux syllabes ne sont pas identiques. La seconde syllabe sáng (matin) avec le ton montant (en caractères gras) reçoit l'accent lexical : c'est un lexème libre (racine). Quant à la première syllabe sang avec le ton plat, c'est un morphème lié (désaccentué), dont la présence permet d'atténuer le sens du lexème libre. Le mot sang sáng signifie donc " au petit matin, à l'aube ». En revanche, les deux syllabes de (4) reçoivent le même ton (ton montant) et l'accent lexical. Il s'agit donc d'une réduplication parfaitement intégrale (consonne initiale, rime et ton). Ces deux syllabes sont des lexèmes libres, dont chacun signifie " matin ", et dont l'association permet d'exprimer l'aspect itératif " tous les matins ».

Rédupliquer des mots à deux syllabes en mots à quatre syllabes est un procédé très fréquemment utilisé à l’oral, selon les quatre modèles suivants (5 à 8). Du point de vue sémantique, les mots à quatre syllabes sont plus forts que ceux à deux syllabes.

(5) hăm hơ (être enthousiaste) $\rightarrow \quad$ hăm hăm hở hơ (être très enthousiaste) 
Dans (5), chacune des deux syllabes constitutives, notées $A B$, est intégralement rédupliquée selon le modèle AABB.

(6) càu nhàu (grommeler) $\rightarrow \quad$ cảu nhảu càu nhàu (grommeler fort)

Dans (6), le mot rédupliqué ( $\mathrm{AB})$ en caractères gras est en position finale, alors que le mot réduplicant (A'B') est en position initiale. Le ton est différent entre le mot rédupliqué et le mot réduplicant. Le modèle est le suivant : A'B'AB.

(7) cong queo (être tortueux) $\rightarrow \quad$ cong queo còng quèo (être très tortueux)

À la différence de (6), le mot rédupliqué ( $\mathrm{AB})$ dans (7) en caractères gras est en position initiale, tandis que le mot réduplicant ( $A^{\prime} B$ ') est en position finale. Seul le ton differe entre ces deux mots, qui suivent le modèle suivant : ABA'B'.

(8) khập khiễng (être boiteux) $\rightarrow$ khập khà $k h a ̣ ̂ p ~ k h i \tilde{e} n g$ (être très boiteux)

La réduplication dans (8) est plus complexe que dans (5, 6 et 7) en ce sens que les deux syllabes constitutives (AB) sont en position finale : elles deviennent la troisième et la quatrième syllabes du mot quadrisyllabique. La première syllabe est identique à la troisième $(\mathrm{A})$. En revanche, seule la consonne initiale de la deuxième syllabe (B') est identique à celle de la quatrième syllabe (B), la rime (noyau vocalique, consonne finale et ton) de ces deux syllabes étant différente. Le modèle de ce type de réduplication est comme suit : $\mathrm{AB}$ 'AB.

\subsection{Complexité au niveau syntaxique et sémantique}

Si un apprenant vietnamien pense que l'apprentissage du chinois ou du thaï est plus aisé que celui du français ou du russe, c'est non parce que le chinois et le thaï sont plus simples objectivement que le français et le russe, mais parce que le chinois, le thaï et le vietnamien partagent plusieurs propriétés syntaxiques et sémantiques.

La complexité au niveau syntaxique et sémantique est exprimée par les quatre points suivants : l'ambivalence de certains lexèmes, le syntagme nominal, le syntagme verbal et la relativisation en vietnamien. 


\subsubsection{Ambivalence de certains lexèmes}

Sapir souligne que dans les langues isolantes, la phrase " est toujours de la première importance, le mot comporte un intérêt moindre ${ }^{20}$ " puisqu'une langue isolante " ne combine pas du tout les concepts en des mots uniques (comme le chinois) ». Quant à Gilbert Lazard ${ }^{21}$, il précise que les fonctions, qui sont remplies par les affixes dans les langues flexionnelles, sont assumées par la syntaxe immédiate dans les langues isolantes.

Si en français la morphologie permet de distinguer nettement les catégories auxquelles appartiennent les quatre lexèmes suivants: le nom (amour), le verbe (aimer), l'adjectif (amoureux) et l'adverbe (amoureusement), en revanche, en vietnamien (ou dans les autres langues isolantes), il existe des lexèmes ambivalents, qui ont besoin d'être placés dans un syntagme ou dans une phrase pour qu'on puisse décider de leur nature nominale ou verbale, ou de leurs fonctions de modifieurs de noms ou de verbes. Ainsi, l'absence de flexions dans les langues isolantes en général, et en vietnamien en particulier, rend plus complexe le classement des parties du discours dans les langues isolantes que dans les langues flexionnelles. Prenons l'exemple du lexème thành công en vietnamien.

(9)

$\begin{array}{lll}\text { Anh ấy } & d a ̃ a & \text { thành công. } \\ 3 \text { SG } & \text { ACCOM } & \text { thành công }\end{array}$

" $\mathrm{Il}$ a réussi »

$\begin{array}{lllll}\text { Bài diễn văn } & \text { của } & \text { anh ấy } & \text { rát } & \text { thành công. } \\ \text { discours } & \text { POss } & 3 S \mathrm{SG} & \text { très } & \text { thành công }\end{array}$

"Son discours est très réussi "

$\begin{array}{lll}\text { (11) Thành công } & \text { của } & d u \text { án } X \\ \text { thành công } & \text { poss } & \text { projet } \mathrm{X}\end{array}$

"La réussite du projet $\mathrm{X}$ »

$\overline{20}$ Edward Sapir, op. cit., p. 90.

21 Gilbert Lazard, "La distinction entre nom et verbe en morphologie et en syntaxe ", Modèles linguistiques, VI, fasc. 1, 1984, p. 29-40. 
$\begin{array}{llll}\text { (12) Bi quyét } & \text { của } & \text { sụ } & \text { thành công. } \\ \text { secret } & \text { POSS } & \text { CL } & \text { thành công }\end{array}$ "Le secret de la réussite»

(13) Một buổi thảo luận un séance discuter thành công

"Une discussion réussie »

$\begin{array}{lllll}\text { (14) Anh ấy } & \text { bảo vệ } & \text { thành công } & \text { luậnán } & \text { tiến sĩ. } \\ \text { 3sG } & \text { soutenir } & \text { thành công } & \text { thèse } & \text { doctorat }\end{array}$ " Il a soutenu avec succès sa thèse de doctorat »

Les six exemples ci-dessus montrent que thành công est un lexème complexe, qui participe tantôt du verbe (dynamique ou statif), tantôt du nom, et qui peut fonctionner comme un modifieur de nom ou de verbe. En effet, dans (9), le pronom de la $3^{\mathrm{e}}$ personne occupe la fonction de sujet, alors que thành công, précédé du marqueur d'accompli đã, fonctionne comme un verbe dynamique. Dans (10), bài diễn văn của anh ấy (son discours) est le sujet, rất thành công (très réussi) est le prédicat. Ce lexème, précédé de l'adverbe de degré rất (très), fonctionne comme un verbe de qualité. Dans (11 et 12), qui sont des syntagmes nominaux, thành công, suivi et précédé du marqueur de possession của (de), fonctionne comme un nom. Dans (13 et 14), thành công fonctionne respectivement comme le modifieur du nom thảo luận (discussion) et celui du verbe bảo vệ (soutenir), correspondant ainsi à un adjectif et à un adverbe en français. En résumé, pour déterminer la catégorie et les fonctions de thành công, on devrait le placer dans un contexte discursif. Il convient de noter que ce même phénomène (ambivalence lexicale) existe également dans d'autres langues isolantes.

2.2.2. Syntagme nominal: examen du nom massifet du classificateur

Le syntagme nominal vietnamien comporte trois éléments dans l'ordre suivant : les éléments prénominaux, le noyau nominal et les éléments postnominaux, présentés au tableau 2. 
Tableau 2

\begin{tabular}{|c|c|c|c|c|c|}
\hline \multicolumn{2}{|c|}{ Éléments prénominaux } & Noyau & \multicolumn{3}{|c|}{ Éléments postnominaux } \\
\hline $\begin{array}{c}\text { Expression } \\
\text { de la } \\
\text { totalité }\end{array}$ & $\begin{array}{c}\text { Expression } \\
\text { de la } \\
\text { quantité }\end{array}$ & CL+N & Sous-classes & Déterminants & Déictiques \\
\hline
\end{tabular}

Les éléments prénominaux (expression de la totalité et expression de la quantité) servent à quantifier le noyau nominal. Quant aux éléments postnominaux (sous-classes, déterminants et déictiques), ils visent à qualifier et à déterminer le noyau. J'ai choisi de souligner la complexité liée au fonctionnement des deux constituants du noyau nominal (nom massif et classificateur) du syntagme nominal.

\section{(a) Noms massifs}

Une autre conséquence directe de l'absence de flexions est que les noms sont d'une forte indétermination ${ }^{22}$, ce qui constitue une principale caractéristique aréale concernant les langues isolantes du Sud-Est de l'Asie ainsi que le chinois : en effet, les noms sans contexte discursif peuvent recevoir plusieurs interprétations. Prenons l'exemple du nom vietnamien thu qui peut signifier « lettre, lettres, une lettre, la lettre, etc. ». Le recours aux classificateurs permet d'individualiser les noms massifs.

En vietnamien, en chinois et dans les autres langues isolantes du Sud-Est de l'Asie, les noms massifs sont en très grande majorité, ils servent à nommer une espèce, une substance ou une qualité. (15) Bốn

con chó

quatre CL (animé) espèce 'chien'

" quatre chien(ne)s"

Si en français et en anglais les noms chien et dog informent du sexe et du nombre (singulier, comptable), en revanche, le lexème vietnamien chó est un nom massif, qui n'informe ni du genre ni du nombre, et qui signifie "espèce 'chien' ». L'emploi du classificateur animé con, antéposé à ce nom, le transforme en nom

22 Voir Walter Bisang, "Classifiers in East and Southeast Asian Languages: Counting and Beyond ", Changes in Numeral Systems, Berlin, Mouton de Gruyter, 1999, p. 113-185. 
comptable, d'où la possibilité d'utiliser le numéral bốn (quatre), comme dans (15). Examinons maintenant les contextes où l'on recourt seulement au nom massif chó (ex. 16 à 21).

Chó lón $\quad$ hơn
espèce 'chien' mèo
"L'espèce 'chien' est plus grande que l'espèce 'chat' "
"Le chien est plus grand que le chat "

$\begin{array}{lllll}\text { Chó } & \text { là } & \text { một } & \text { loài dộng vật. } \\ \text { espèce 'chien' } & \text { COP } & \text { un } & \text { espèce } & \text { animal }\end{array}$

"L'espèce 'chien' est une espèce animale "

$\begin{array}{llll}\text { Tồi thích chó } & \text { hơn } & \text { mèo. } \\ \text { ISG aimer } & \text { espèce 'chien' } & \text { dépasser } & \text { espèce 'chat' }\end{array}$

"Je préfère les chiens aux chats"

Chó thì có chó dục, chó cái, chó con.
espèce 'chien' Top avoir espèce 'chien' mâle espèce 'chien' femelle espèce 'chien' enfant "Quant à l'espèce 'chien', il y a des chiens, des chiennes et des chiots »
Ho $\quad \breve{a n} o^{\circ}$
$n h u$
chó
với
mèo.
$3 \mathrm{PL}$ se comporter comme espèce 'chien' avec espèce 'chat'
"Ils se comportent comme chien et chat »

Chó nhà nó sủa suốt cả ngày.
chien maison 3sG aboyer tout jour
"Ses chiens (son chien) ont (a) aboyé toute la journée"

Le fonctionnement du lexème chó est complexe, car il peut être utilisé dans de nombreux contextes discursifs comme en témoignent les exemples (16 à 21). On ne recourt au nom massif que lorsqu'on procède à une comparaison entre deux espèces (ex. 16), à une identification (ex. 17). Dans (18), le locuteur fait part de son affection pour l'espèce 'chien' plutôt que pour l'espèce 'chat'. Quant à (19), il illustre la relation « hyperthème - thème " : le nom massif chó, qui représente une espèce animale, en position initiale, est thématisé par le marqueur thi. Les sous-classes de cette espèce sont placées dans le rhème (après le thématiseur thi). L'exemple (20) est une expression idiomatique figée où le locuteur ne réfère ni à un chien ni à un chat précis. 
Le contexte de (21) diffère complètement de celui de (16 à 20) en ce sens que chó ne réfere pas à l'espèce 'chien' (valeur générique), mais à une valeur spécifique : le locuteur désigne les chiens (ou le chien) de son voisin.

\section{(b) Classificateurs}

McWhorter ${ }^{23}$ souligne que le chinois est plus complexe que les langues créoles du fait que le premier est une langue à classificateurs, alors que les secondes sont des langues sans classificateur. Pourquoi l'emploi des classificateurs peut-il rendre une langue complexe au niveau sémantico-syntaxique?

Dans son article intitulé "Classifiers in East and Southeast Asian Languages: counting and beyond ", Bisang ${ }^{24}$ souligne que les classificateurs dans les langues du Sud-Est de l'Asie peuvent avoir trois fonctions principales : celle de "classification ", celle

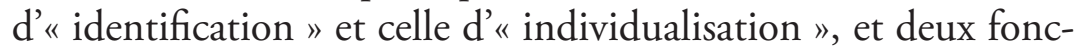
tions dérivées : celle de "référentialisation » et celle de "relationalisation ». L'auteur précise d'ailleurs qu'il existe une hiérarchie entre les trois fonctions essentielles comme suit : la classification est la condition préalable pour l'identification ${ }^{25}$. L'identification peut s'opérer sans avoir à référer à l'individualisation. Ces trois fonctions sont récapitulées dans la figure ci-après :

Classification >

Identification >

Individualisation

Référentialisation

Relationalisation

(22)

$\begin{array}{lllll}\text { Đua } & c h o & \text { tôi } & c a ́ i & a ́ o ! \\ \text { passer } & \text { donner } & \text { ISG } & \text { CL (inanimé) } & \text { chemise }\end{array}$

« Passe-moi la chemise! "

$\begin{array}{lcll}\text { Cái } & \text { áo } & \text { của } & \text { Jean... } \\ \text { CL(inanimé) } & \text { chemise } & \text { poss } & \text { Jean } \\ \text { "La chemise de Jean... " } & & \end{array}$

\footnotetext{
23 John Hamilton McWhorter, op. cit., p. 156.

24 Walter Bisang, op. cit., 1999, p. 116.

25 Voir William Croft, "Semantic Universals in Classifier Systems ", Word, vol. 45, n 2, 1994, p. 161.
} 
(24)

$\begin{array}{llllll}\text { Cái } & a ́ o & \text { (mà) } & \text { Jean } & \text { mua } & \text { hôm qua... } \\ \text { CL (inanimé) } & \text { chemise } & \text { ReL } & \text { Jean } & \text { acheter } & \text { hier }\end{array}$

"La chemise que Jean a achetée hier... "

En vietnamien, les fonctions de « classification " et d' " individualisation » du classificateur sont illustrées par (15) : con est un classificateur pour objet animé (fonction de " classification »), son emploi permet de rendre comptable le lexème chó (fonction $\mathrm{d}^{\prime}$ " individualisation »). Quant à la fonction d' "identification ", elle est illustrée à travers ses fonctions dérivées "référentialisation " et " relationalisation ", rencontrées dans (22, 23 et 24). L'exemple (22) présente un contexte déictique : le référent nominal est d'emblée identifié et défini par l'emploi du classificateur pour objet inanimé cái. Autrement dit, (22) n'est utilisé que lorsque le locuteur et l'allocutaire savent de quel objet il s'agit (fonction de "référentialisation"). Quant à (23 et 24), ce sont des syntagmes nominaux étendus, dans lesquels l'objet est identifié grâce à sa relation avec le syntagme prépositionnel của Jean (de Jean) ou avec la proposition relative mà Jean mua hôm qua (que Jean a achetée hier). Ces deux exemples sont respectivement une structure possessive et une structure relative (fonction de "relationalisation "). Dans les contextes de (22, 23 et 24), le classificateur cái est rendu par les déterminants articles définis le en français et the en anglais.

\subsubsection{Syntagme verbal : examen du verbe di}

Le syntagme verbal vietnamien comporte trois éléments dans l'ordre suivant : les éléments préverbaux, le noyau verbal et les éléments postverbaux, présentés au tableau 3.

Tableau 3

\begin{tabular}{|c|c|c|c|c|}
\hline Éléments préverbaux & Noyau verbal & \multicolumn{3}{|c|}{ Éléments postverbaux } \\
\hline Marqueurs prédicatifs & $\begin{array}{c}\text { un verbe ou } \\
\text { une CVS }\end{array}$ & aspect & objet & Marqueurs prédicatifs \\
\hline
\end{tabular}


Les éléments préverbaux (marqueurs prédicatifs) et les éléments postverbaux (aspect, objet et marqueurs prédicatifs) servent à exprimer le TAM (temps, aspect, modalité), la fréquence, l'intensité, les modalités du noyau verbal, qui peut être composé d'un seul verbe ou d'une série de verbes appelée construction verbale en série (CVS).

J'ai choisi d'examiner la construction verbale en série au moyen du verbe di (aller) pour illustrer un autre aspect de complexité. En effet, ce morphème peut avoir plusieurs fonctions lorsqu'il est placé dans un énoncé. En tant que lexème, di est un verbe de mouvement. Grammaticalisé, di fonctionne comme un marqueur impératif. Pragmaticalisé, il devient un marqueur pragmatique traduisant l'attitude du locuteur à l'égard de l'allocutaire. Le chemin d'évolution sémantique de đi peut être récapitulé au tableau 4.

Tableau 4

\begin{tabular}{|l|l|l|}
\hline \multicolumn{1}{|c|}{ Unité lexicale } & \multicolumn{1}{c|}{ Unité grammaticale } & \multicolumn{1}{c|}{ Unité pragmatique } \\
\hline $\begin{array}{l}\text { di en tant que } \\
\text { verbe de mouvement }\end{array}$ & $\begin{array}{l}\text { di en tant que } \\
\text { marqueur impératif }\end{array}$ & $\begin{array}{l}\text { di en tant que } \\
\text { marqueur discursif final }\end{array}$ \\
\hline
\end{tabular}

(a) Le verbe di en tant que lexème

En tant que lexème, $d i$, verbe de mouvement centrifuge, est utilisé dans de nombreuses compositions lexicales, où il est en première position : đi + verbe.

$$
\begin{array}{ll}
\text { di } & \text { díng } \\
\text { aller } & \text { être debout }
\end{array}
$$

"se comporter"

(26) di lại

aller venir

"se déplacer»

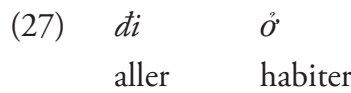

"se faire domestique " 
Dans (25, 26 et 27), di (aller) se combine avec díng (être debout), lại (venir), $o^{\prime}$ (habiter) pour former des CVS, dont le sens diffère de celui de chacun des deux composants. Sémantiquement, aucun de ces deux composants ne prévaut l'un sur l'autre. Par conséquent, ils reçoivent tous l'accent lexical.

$$
\begin{aligned}
& \text { di } \quad \text { boc } \\
& \text { aller apprendre } \\
& \text { "aller à l'école " } \\
& \text { di } \quad b o r i \\
& \text { aller nager } \\
& \text { "aller à la piscine " }
\end{aligned}
$$

(29) di boi

Dans (28 et 29), le $\mathrm{V}_{1}$ exprime un mouvement centrifuge, alors que le $\mathrm{V}_{2}$ indique le but de ce mouvement. Le rapport entre ces deux verbes est déterminé-déterminant. Par conséquent, seul le $\mathrm{V}_{2}$ est accentué, et suit chronologiquement le $\mathrm{V}_{1}$.

$\begin{array}{lll}\text { di } \quad \text { hoc } & \text { về } \\ \text { aller apprendre } & \text { rentrer } \\ \text { "rentrer de l'école " } & \end{array}$

di boi vì
aller nager rentrer
"rentrer de la piscine "

À partir de (28 et 29), on peut avoir une série de trois verbes comme dans (30 et 31 ), où le $V_{3}$ indique un mouvement centripète : on revient au point de départ. L'ordre de ces trois verbes constitutifs est chronologique ou $"$ iconique $»^{26}$.

$$
\begin{aligned}
& \text { di lên } \\
& \text { aller monter } \\
& \text { "monter " } \\
& \text { "go up " }
\end{aligned}
$$

26 Alexandra Y. Aikhenvald et R. M. W. Dixon (dir.), Serial Verb Constructions: A Cross-linguistic Typology, Oxford, Oxford University Press, 2006. 
(33)

$$
\begin{array}{ll}
\text { di } & \text { xuống } \\
\text { aller } & \text { descendre }
\end{array}
$$

"descendre "

" go down"

$$
\begin{aligned}
& \text { di ra } \\
& \text { aller sortir } \\
& \text { "sortir" } \\
& \text { "go out" }
\end{aligned}
$$

$$
\begin{array}{ll}
\text { di } & \text { vào } \\
\text { aller } & \text { entrer }
\end{array}
$$

« entrer»

" go in "

Les exemples (32 à 35) désignent l'expression de la trajectoire : les $V_{2}$ indiquent la direction. Comme dans (28 et 29), ces constructions illustrent également un rapport déterminé-déterminant. Seuls les $\mathrm{V}_{2}$ sont donc accentués.

L'examen de (25 à 35) conduit aux quelques remarques suivantes : ces exemples illustrent ce qu'on appelle des constructions verbales en série (ou CVS), une caractéristique aréale relative aux langues isolantes du Sud-Est de l'Asie ainsi qu'au chinois. D'après Alexandra Y. Aikhenvald et R.M.W. Dixon, les CVS décrivent :

[...] ce qui correspond conceptuellement à un événement unique. Les verbes d'une CVS partagent les informations de temps, d'aspect, de modalité et de polarité; ils ont au moins un argument en commun, et ne peuvent avoir qu'un seul sujet. Aucun des verbes de la CVS n'appartient à une proposition subordonnée. L'intonation de la CVS est celle d'une proposition unique ${ }^{27}$.

Les exemples (32 à 35) illustrent bien les différences entre le vietnamien, le français et l'anglais dans l'expression de la trajectoire. En vietnamien, langue " equipollently-framed $»^{28}$, le mouvement et la

Alice Vittrant, «Les constructions de verbes en série : une autre approche du syntagme verbal en birman ", Bulletin de la Société de Linguistique de Paris, tome CI, fasc. 1, 2006, p. 309.

Voir Daniel Isaac Slobin, «The Many Ways to Search for a Frog: Linguistic Typology and the Expression of Motion Events ", dans Sven Strömqvist et Ludo Th. Verhoeven (dir.), Relating events in narrative, vol. 2, Typological and contextual perspectives, Mahwah NJ, Lawrence Erlbaum, 2004, p. 219-257. 
direction sont traduits par deux verbes ayant le même poids sémantique. En anglais, langue "satellite-framed " ${ }^{29}$, le premier est indiqué par un verbe, la seconde par un adverbe. Quant au français, langue "verb-framed", ceux-ci sont encodés par un seul verbe.

$\begin{array}{llll}\text { Mang } & \text { di } & \text { Chay } & d i \\ \text { porter } & \text { aller } & \text { courrir } & \text { aller } \\ \text { "Emporter " } & \text { "Courir vers un autre lieu " } & \\ \text { "Take away " } & \text { "Run away " }\end{array}$

$\begin{array}{llll}\text { Nhin } & \text { di } & \text { chố } & \text { khác. } \\ \text { regarder } & \text { aller } & \text { place } & \text { être différent }\end{array}$

« Diriger son regard vers un autre endroit »

(38) Anhốy dã mang di tất cả các cuốn sách này. 3sg ACCOM porter aller tout pluriel CL livre DEICT "Il emporte tous ces livres"

Le verbe di entre aussi dans les compositions lexicales, où il est en deuxième position (verbe + di) comme dans (36, 37 et 38). Dans cette position, $d i$, verbe de mouvement centrifuge, sert à déplacer la direction initiale exprimée par les $\mathrm{V}_{1}$, qui sont des verbes d'activités : mang (porter), chạy (courir), nhin (regarder). Les deux verbes $\left(\mathrm{V}_{1}\right.$ et $\left.\mathrm{V}_{2}\right)$ sont tous accentués. L'emploi de di correspond à celui du préfixe français en- et à l'adverbe anglais away.
(39) Anh ấy gầy
di (già di) (xấu
di)
3SG être maigre aller être vieux aller être laid aller
"Paul maigrit (vieillit) (enlaidit)"

À la différence de (36 à 38), lorsque di est postposé aux verbes d'état (être maigre, être vieux, être laid) de (39), il transforme ces états en processus (maigrir, vieillir, enlaidir), et traduit donc l'aspect inchoatif.

(b) Le verbe di en tant que grammème

29 Leonard Talmy, "Lexicalization Patterns: Semantic Structure in Lexical Forms ", dans Timothy Shopen (dir.), Language typology and syntactic description, vol. 3, Cambridge, Cambridge University Press, 1985, p. 36-149. 
Dans les exemples ci-dessous, di ne fonctionne plus comme un verbe (lexème), mais comme un marqueur impératif (grammème).
(40) uống di!
boire IMP
«buvez! »
(41) $\breve{a n}$
$d i !$
manger IMP
« mangez! »
(42) di di!
aller IMP
« allez-vous en! »

Le mot di ne fonctionne comme un marqueur impératif que lorsqu'il est précédé de verbes d'activité et ne reçoit pas l'accent lexical, comme dans $(40,41$ et 42$)$, à la différence de đi accentué dans (36, 37 et 38). Notons que dans (42), di (aller) en tant que verbe s'utilise sans ambiguïté avec di en tant que marqueur impératif. La grammaticalisation de di est achevée, puisque l'unité source (verbe) et l'unité cible (marqueur impératif) peuvent se côtoyer dans un même énoncée ${ }^{30}$.
(43) Anh mang di tất cả các cuốn sách này di! 2SG porter aller tout pluriel CL livre DEICT IMP "(Svp) Emportez tous ces livres! »

(44) Lấy cái áo này di! prendre CL chemise DEICT IMP «Prenez cette chemise! »
(45) Lấy cái áo này di di! prendre CL chemise DEICT aller IMP «Emportez cette chemise! »

Les exemples (43 et 45) sont respectivement plus complexes que (38 et 44) en raison de la présence de deux di, l'un est interprété comme verbe, l'autre comme marqueur impératif.

30 Voir le principe d' " anachronie » dans Claude Hagège, The Language Builder, Amsterdam-Philadelphia, John Benjamins Publishing Company, 1993. 
Cependant, (38) diffère de (43) en ce que (38) est une assertion où di est identifié comme verbe, donc accentué, tandis que (43) est une injonction où di fonctionne comme un marqueur impératif, donc désaccentué.

\subsubsection{Relativisation : examen du marqueur mà}

Claire Martinot ${ }^{31}$ souligne que, en général, plus la syntaxe est intégrée, plus la complexité est grande, et que, en théorie, le degré de complexité progresse dans l'ordre suivant : la juxtaposition < la coordination < la subordination < la prédication complexe. J'ai choisi d'étudier le relativiseur mà dans les structures de subordination de (46 à 49).

(46) Người đàn ông $\emptyset$ homme $\begin{array}{lllll}\text { dang } & \text { viét } & \text { là } & \text { thầy } & \text { tôi. } \\ \text { PROG } & \text { écrire } & \text { COP } & \text { professeur } & \text { ISG }\end{array}$ "L'homme qui est en train d'écrire est mon professeur "

Cuốn sách Ø/ mà tôi mua hômqua rất hay. CL livre mà ISG acheter hier très être intéressant "Le livre que j'ai acheté hier est très intéressant "

(48) Đứa bé mà / Ø tôi cho sách hồi nãy là cháu tôi. CL enfant mà ISG donner livre tout à l'heure COP neveu ISG "L'enfant à qui j'ai donné un livre tout à l'heure est mon neveu "

(49) Đứa bé mà chiếcxedạp bịhu là cháu tôi. CL enfant mà CL bicyclette être en panne Cop neveu 1sG "L'enfant dont la bicyclette est en panne est mon neveu »

Delle Luche et ses collaborateurs ${ }^{32}$ précisent que la relativisation est une source de complexité d'ordre syntaxique et cognitif, et qu'une relativisation du sujet, avec l'ordre canonique sujetverbe-objet, est moins complexe qu'une relativisation de l'objet.

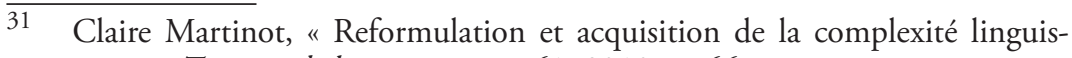
tique ", Travaux de linguistique, n 61, 2010, p. 66.

32 Claire Delle Luche et al., "Syntaxe et sémantique dans la compréhension de propositions relatives en français ", communication au colloque international des étudiants chercheurs en didactique des langues et en linguistique, Grenoble, 4-7 juillet 2006. 
Cela rejoint parfaitement le point de vue de Keenan et Comrie ${ }^{33}$ et celui de Amara et Natchanan ${ }^{34}$, selon qui le sujet est plus accessible à la relativisation que l'objet direct, qui est plus accessible à la relativisation que l'objet indirect, qui est plus accessible à la relativisation que le possesseur.

Les exemples (46 à 49) illustrent respectivement une relativisation du sujet (46), de l'objet direct (47), de l'objet indirect (48) et du possesseur (49). La présence ou l'absence du relativiseur mà dépend du degré de complexité selon l'ordre suivant : le sujet < l'objet direct < l'objet indirect < le possesseur. En cas de relativisation du sujet (46), l'absence de mà est nécessaire. En (47), la présence de ce marqueur n'est pas nécessaire, à la différence de (48), où sa présence est nécessaire. En (49), mà est indispensable. Autrement dit, la présence du relativiseur mà est obligatoire dans le cas le plus complexe (49).

\subsection{Complexité au niveau discursif}

Le vietnamien, tout comme les autres langues isolantes du Sudest de l'Asie, dispose de très nombreuses particules placées en fin d'énoncé (appelées " particules discursives finales » ou " attitudinal sentence-final particles ", selon Li et collaborateurs ${ }^{35}$ ), exprimant soit les différentes attitudes du locuteur, soit les différents types de phrase (interrogative, impérative, exclamative). La présence de ces particules finales constitue indéniablement un cas complexe dans le fonctionnement des langues du Sud-est en général, et du vietnamien en particulier. Ces particules, très fréquemment utilisées dans la conversation quotidienne, les dialogues de romans ou les interviews, ne trouvent pas leurs équivalentes en anglais ou en français. Cela représente une source de difficultés

\footnotetext{
33 Edward Keenan et Bernard Comrie, "Noun Phrase Accessibility and Universal Grammar ", Linguistic Inquiry n 8, 1977, p. 63-99.

34 Prasithrathsint Amara et Yaowapat Natchanan, "A Typology of Relative Clauses in Mainland Southeast Asian Languages ", Mon-Khmer Studies Journal (Thailand), $\mathrm{n}^{\circ} 38,2008$, p. 9.

35 Charles N. Li, R. McMillan Thompson and Sandra A. Thompson, "The Discourse Motivation for the Perfect Aspect, the Mandarin Particle le », dans Paul J. Hopper (dir.), Tense and Aspect, Amsterdam, John Benjamins, 1982, p. 21.
} 
dans l'apprentissage des langues isolantes par les apprenants de langues indo-européennes. Examinons maintenant le cas de di et de $m \dot{a}$.

(50)

$\begin{array}{lllll}\text { Thích } & q u a ́ & d i & c h u ́ & m \dot{a} ! \\ \text { adorer } & \text { PF } & \text { PF } & \text { PF } & \text { PF } \\ \text { "Combien j'adore ça!" } & & \end{array}$

(51) $R \tilde{a}$

$\begin{array}{lllllllll}R o ́ & q u a ́ & \text { đi } & \text { rồi } & \text { mà! } & \text { Anh còn kêu ca } & \text { gì nữa? } \\ \text { être clair } & \text { PF } & \text { PF } & \text { PF } & \text { PF } & \text { 2SG } & \text { encore se plaindre } & \text { quoi encore }\end{array}$

"C'est tellement clair (maintenant)! » «De quoi te plains-tu? »

(52) Mẹ dã bảo rồi mà!

maman ACCOM dire $P F \quad P F$

«Maman te l'avait dit! »

(53) Trời ởi Tồi tuớng anh di rồi kia mà!

Dieu oh 1SG penser 2SG partir PF $\quad$ PF $\quad$ PF

«Mon Dieu! Je pensais que tu étais parti!»

Le marqueur di dans (50 et 51) et le marqueur mà dans (50 à 53) fonctionnent comme des particules discursives finales (appelées aussi des pragmatèmes), fréquemment utilisées à l'oral pour traduire les attitudes du locuteur. En tant que particules finales, đi et mà sont des éléments externes aux énoncés, et ont un rôle extraphrastique. Ils ne participent plus à la construction du sens référentiel des énoncés, mais représentent des traces de l'intervention du locuteur. On peut dire que $d i$ dans (50 et 51) et mà dans (50 à 53) sont le "véhicule par excellence du regard $d u$ locuteur " ${ }^{36}$ à l'égard de l'allocutaire.

La multitude des particules finales en vietnamien est certes une source de complexité, mais l'ordre de ces particules finales en constitue une autre source dans l'apprentissage du vietnamien. Dans (50 et 51), quá (particule d'exclamation) doit être immédiatement suivie de $d i$ (particule de renforcement). La particule mà est toujours placée en fin d'énoncé comme dans (50 à 53). On peut placer, entre di et $m \dot{a}$, soit une particule de renforcement à valeur affirmative comme chú en (50), soit une particule à

36 Voir Henning Nølke, Le regard du locuteur, Paris, Kimé, 1993. 
valeur accomplie comme rồi en (51). Entre rồi et mà, on peut insérer une particule de renforcement comme kia en (53).

Du point de vue sémantique, la particule di permet au locuteur de renforcer son adhésion au contenu de l'énoncé, comme dans (50 et 51). Quant à mà, c'est une particule dont le locuteur se sert pour exprimer ses sentiments (irritation, avertissement, reproche, surprise, explication...) à l'égard de l'allocutaire. Sans cette particule, (52 et 53) sont interprétés comme des énoncés assertifs à valeur descriptive. Avec mà, (52) est un reproche de la mère adressée à son enfant, alors que (53) traduit une surprise du locuteur.

\section{Conclusion}

Je pense que toutes les langues sont aussi complexes les unes que les autres, mais je n'adhère pas entièrement à l'hypothèse compensatoire (soutenue par Riddle et bien d'autres linguistes), selon laquelle si les langues isolantes sont plus simples morphologiquement, elles sont, par compensation, plus complexes du point de vue syntaxique et sémantique. Cette hypothèse est, me semblet-il, relativement réductrice puisqu'elle présuppose que la morphologie des langues isolantes doit être plus simple que celle des langues flexionnelles, et que la syntaxe des langues isolantes doit être, par compensation, plus complexe que celle des langues flexionnelles. En réalité, la complexité des langues du monde n'est pas répartie de façon systématique comme le suggère cette hypothèse. Je suis persuadé que la syntaxe des langues isolantes n'est pas plus complexe que celle des langues flexionnelles, et inversement la morphologie des langues isolantes n'est pas plus simple que celle des langues flexionnelles. Le problème est qu'il faudrait comparer ce qui est comparable. Du point de vue morphologique, il convient de distinguer deux types de morphologie : la morphologie flexionnelle et la morphologie lexicale. Certes pour le premier type de morphologie (morphologie flexionnelle), les langues isolantes, dépourvues de flexions, doivent être logiquement et théoriquement plus simples que les langues flexionnelles. Mais, pour le second type de morphologie 
(morphologie lexicale), les langues isolantes disposent de mécanismes comme la réduplication (consonne initiale, rime...) et le jeu des tons considérés comme très complexes.

Je précise en outre qu'on ne peut mesurer efficacement les degrés de complexité entre deux ou plusieurs langues que si celles-ci appartiennent au même type de langues : type isolant (vietnamien, chinois, thaï...), type flexionnel (français, italien, espagnol, anglais...), etc. Mais, on devrait être plus nuancé lorsqu'il s'agit de comparer deux langues de types différents (vietnamien et français). Autrement dit, pour remettre en question l'hypothèse compensatoire, je me suis attaché à montrer, dans cet article, que la complexité du vietnamien, langue isolante, peut être résumée aux trois principaux niveaux suivants : le niveau morpho-phonologique, le niveau syntactico-sémantique et le niveau discursif.

$\mathrm{Au}$ niveau morpho-phonologique, le vietnamien est une langue isolante, riche de six tons, dont l'apparition s'est produite au fil des siècles. La réduplication, caractéristique des langues du Sud-Est de l'Asie, participant à la formation des mots, est très productive : elle est formée par le jeu des tons et des consonnes initiales et des rimes.

Au niveau syntactico-sémantique, en l'absence de morphologie flexionnelle, on constate une grande indétermination lexicale : certains lexèmes, ambivalents, peuvent avoir plusieurs fonctions (nom, verbe, modifieur de nom, modifieur de verbe) selon leur place dans le syntagme. Le nom massif peut être utilisé dans plusieurs contextes, de même que le classificateur possède plusieurs fonctions (classification, individualisation, référentialisation et relationalisation).

La polycatégorialité, source de complexité, a été illustrée par les mots di et mà qu'on peut rencontrer aussi bien au niveau syntaxique qu'au niveau discursif. Le verbe de mouvement $d i$ (lexème) peut fonctionner dans un autre contexte comme un marqueur impératif (grammème) ou une particule discursive (pragmatème). Quant à mà, il peut être utilisé, soit comme un relativiseur, soit comme une particule finale à sentiments (pragmatème). 


\section{Abréviations}

ISG ( $1^{\mathrm{e}}$ personne du singulier); 2SG ( $2^{\mathrm{e}}$ personne du singulier); 3 SG ( $3^{\mathrm{e}}$ personne du singulier); 3 PL ( $3^{\mathrm{e}}$ personne du pluriel); ACCOM (accompli); CL (classificateur); COP (copule); DEICT (déictique); IMP (impératif); PF (particule finale); POSs (marqueur de possession); REL (relavitiseur); TOP (topicaliseur). 


\section{Bibliographie}

Aikhenvald, Alexandra Y. et R. M. W. Dixon (dir.), Serial Verb Constructions: A Cross-linguistic Typology, Oxford, Oxford University Press, 2006.

Aitchison, Jean, Language Change: Progress or Decay? Cambridge, Cambridge University Press, 1991.

Amara, Prasithrathsint et Yaowapat Natchanan, «A Typology of Relative Clauses in Mainland Southeast Asian Languages ", Mon-Khmer Studies Journal (Thailand), n 38, 2008, p. 1-23.

Bisang, Walter, "On the Evolution of Complexity - Sometimes Less is More in East and Mainland Southeast Asian ", dans Geoffrey Sampson, David Gil, Peter Trudgill (dir.), Language Complexity as an Evolving Variable, Oxford, Oxford University Press, 2009, p. 34-49.

Bisang, Walter, "Classifiers in East and Southeast Asian Languages: Counting and beyond ", Changes in Numeral Systems, Berlin, Mouton de Gruyter, 1999, p. 113-185.

Coyos, Jean-Baptiste, "La langue basque selon Gustave Guillaume : quelques commentaires d'un point de vue structuraliste fonctionnaliste ", dans les Actes du XXe Colloque international de psychomécanique du langage, Oloron-Sainte-Marie (France), 2004, p. 289-303.

Croft, William, "Semantic Universals in Classifier Systems », Word, vol. 45, $\mathrm{n}^{\circ} 2$, 1994, p. 145-171.

Dahl, Östen, The Growth and Maintenance of Linguistic Complexity, Amsterdam, Benjamins, 2004.

Delle Luche, Claire, "Syntaxe et sémantique dans la compréhension de propositions relatives en français ", communication au colloque international des étudiants chercheurs en didactique des langues et en linguistique, Grenoble, 4-7 juillet 2006.

Fenk-Oczlon, Gertraud et August Fenk, « Complexity Trade-Offs Between the Subsystems of Language », dans Matti Miestamo, Kaius Sinnemäki et Fred Karlsson (dir.), Language Complexity: Typology, Contact, Change, Amsterdam, Benjamins, 2008, p. 43-65.

Gil, David, "How Complex are Isolating Languages », dans Matti Miestamo, Kaius Sinnemäki, Fred Karlsson (dir.), Language Complexity: Typology, Contact, Change, Amsterdam, Benjamins, 2008, p. 109-131.

Glaudert, Nathalie, La complexité linguistique : essai de théorisation et d'application dans un cadre comparatiste, Thèse de doctorat, Université de la Réunion, 2011.

Hagège, Claude, The Language Builder, Amsterdam-Philadelphia, John Benjamins Publishing Company, 1993. 
Haudricourt, Georges, "De l'origine des tons en vietnamien ", Journal Asiatique, no 242, 1954, p. 69-82.

Hockett, Charles Francis, A Course in Modern Linguistics, New York, Macmillan, 1958.

Keenan, Eward et Bernard Comrie, "Noun Phrase Accessibility and Universal Grammar », Linguistic Inquiry n 8, 1977, p. 63-99.

Kusters, Wouter, "Complexity in Linguistic Theory, Language Learning and Language Change ", dans Matti Miestamo, Kaius Sinnemäki, Fred Karlsson (dir.), Language Complexity: Typology, Contact, Change, Amsterdam, Benjamins, 2008, p. 3-22.

Lazard, Gilbert, « La distinction entre nom et verbe en morphologie et en syntaxe ", Modèles linguistiques, VI, fasc. 1, 1984, p. 29-40.

Li, Charles N., R. McMillan Thompson et Sandra A. Thompson, «The Discourse Motivation for the Perfect Aspect, the Mandarin Particle le ", dans Paul J. Hopper (dir.), Tense and Aspect, Amsterdam, John Benjamins, 1982, p.19-44.

Martinot, Claire, "Reformulation et acquisition de la complexité linguistique ", Travaux de linguistique, $\mathrm{n}^{\circ}$ 61, 2010, p. 63-96.

McWhorter, John Hamilton, "The World’s Simplest Grammars are Creole Grammars », Linguistic Typology n ${ }^{\circ}$ 5, 2001, p. 125-166.

Miestamo, Matti, "Grammatical Complexity in Cross-Linguistic Perspective », dans Matti Miestamo, Kaius Sinnemäki et Fred Karlsson (dir.), Language Complexity: Typology, Contact, Change, Amsterdam, Benjamins, 2008, p. 23-41.

Nguyên, Phu Phong, Le syntagme verbal en vietnamien, Paris, Mouton, La Haye, 1976.

Nølke, Henning, Le regard du locuteur, Paris, Kimé, 1993.

Paolo, Ramat, « De la complexité des langues. À propos de l'axiome 'ALEC' (All Languages are Equally Complex) ", communication à la journée d'étude La nature de la complexité linguistique, organisée par Claire Martinot, 2 et 3 juillet 2012, Maison de la recherche, Paris.

Riddle, Elizabeth M., "Complexity in Isolating Languages: Lexical Elaboration Versus Grammatical Economy ", dans Matti Miestamo, Kaius Sinnemäki, Fred Karlsson (dir), Language Complexity: Typology, Contact, Change, Amsterdam, Benjamins, 2008, p. 133-151.

Sapir, Edward, Language: An Introduction to the Study of Speech, New York, Harcourt, Brace (Le langage. Introduction à l'étude de la parole, traduit de l'anglais par Guillemin), 1921.

Shosted, Ryan, "Correlating Complexity: A Typological Approach ", Linguistic Typology 10, 2006, p. 1-40. 
Slobin, Daniel Isaac, "The Many Ways to Search for a Frog: Linguistic Typology and the Expression of Motion Events ", dans Sven Strömqvist et Ludo Th. Verhoeven (dir,), Relating Events in Narrative, vol. 2, Typological and Contextual Perspectives, Mahwah NJ, Lawrence Erlbaum, 2004, p. 219-257.

Talmy, Leonard, "Lexicalization Patterns: Semantic Structure in Lexical Forms ", dans Timothy Shopen (dir.), Language Typology and Syntactic Description, vol. 3, Cambridge, Cambridge University Press, 1985, p. 36-149.

Vittrant, Alice, «Les constructions de verbes en série : une autre approche du syntagme verbal en birman ", Bulletin de la Société de Linguistique de Paris, tome CI, fasc. 1, 2006, p. 305-367. 